\title{
Astrocyte signaling in the presence of spatial inhomogeneities
}

\author{
Michail Stamatakis and Nikos V. Mantzaris ${ }^{\text {a) }}$ \\ Department of Chemical and Biomolecular Engineering, Rice University, Houston, Texas 77005, USA
}

(Received 21 February 2007; accepted 10 July 2007; published online 27 September 2007)

\begin{abstract}
Astrocytes, a special type of glial cells, were considered to have just a supporting role in information processing in the brain. However, several recent studies have shown that they can be chemically stimulated by various neurotransmitters, such as ATP, and can generate $\mathrm{Ca}^{2+}$ and ATP waves, which can propagate over many cell lengths before being blocked. Although pathological conditions, such as spreading depression and epilepsy, have been linked to abnormal wave propagation in astrocytic cellular networks, a quantitative understanding of the underlying characteristics is still lacking. Astrocytic cellular networks are inhomogeneous, in the sense that the domain they occupy contains passive regions or gaps, which are unable to support wave propagation. Thus, this work focuses on understanding the complex interplay between single-cell signal transduction, domain inhomogeneity, and the characteristics of wave propagation and blocking in astrocytic cellular networks. The single-cell signal transduction model that was employed accounts for ATP-mediated $\mathrm{IP}_{3}$ production, the subsequent $\mathrm{Ca}^{2+}$ release from the ER, and ATP release into the extracellular space. The model is excitable and thus an infinite range of wave propagation is observed if the domain of propagation is homogeneous. This is not always the case for inhomogeneous domains. To model wave propagation in inhomogeneous astrocytic networks, a reaction-diffusion framework was developed and one-gap as well as multiple-gap cases were simulated using an efficient finiteelement algorithm. The minimum gap length that blocks the wave was computed as a function of excitability levels and geometric characteristics of the inhomogeneous network, such as the length of the active regions (cells). Complex transient patterns, such as wave reflection, wave trapping, and generation of echo waves, were also predicted by the model, and their relationship to the geometric characteristics of the network was evaluated. Therefore, the proposed model can help in the formulation of testable hypotheses to explain the observed abnormal wave propagation in pathological situations. (C) 2007 American Institute of Physics. [DOI: 10.1063/1.2767409]
\end{abstract}

\section{INTRODUCTION}

The nervous system consists of two types of cells: the neurons, which are responsible for signal propagation and information processing, and the glial cells, which exist in between the neurons. ${ }^{1}$ Our study focuses on a special type of glial cells, the so-called astrocytes. Before 1990, astrocytes were considered to have just a supporting role for the neurons, by holding them together and supplying nutrients to them. However, several recent studies have established that astrocytes play an active role in signal transduction in the synapse, and that extracellular ATP acts as a messenger in this process (see Refs. 2 and 3 for excellent reviews). Thus, changes in intracellular $\mathrm{Ca}^{2+}$ concentration have been observed to result from stimulation with ATP, ${ }^{4}$ and ATP secretion has been shown to follow intracellular $\mathrm{Ca}^{2+}$ increase. ${ }^{5}$

Moreover, it has been shown that the result of the stimulation does not remain confined in one cell, but rather propagates as a wave throughout the astrocytic network. $\mathrm{Ca}^{2+}$ as well as ATP waves have been monitored experimentally. Such waves can result from the application of an extracellular messenger such as glutamate ${ }^{6-9}$ or ATP. ${ }^{10,11}$ Wave propagation can also result from mechanical ${ }^{12-19}$ or electrical

\footnotetext{
${ }^{\text {a) }}$ Author to whom correspondence should be addressed. Telephone: +1-713348-2955; Fax: +1-713-348-5478. Electronic mail: nman@rice.edu
}

stimuli. ${ }^{5,20-22}$ Astrocytic $\mathrm{Ca}^{2+}$ waves propagate with speeds that vary from 10 to $30 \mu \mathrm{m} / \mathrm{s}$ (Refs. 11, 15, and 23-25) across several cell diameters, typically in the order of $\sim 360 \mu \mathrm{m}^{5}$

Even more interestingly, these waves have been shown to be able to cross cell-free zones, indicating that $\mathrm{Ca}^{2+}$-dependent release of an extracellular messenger may be

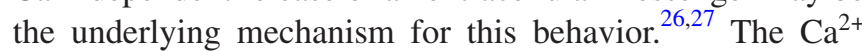
wave can pass between cell cultures separated by cell-free lanes up to $\sim 120 \mu \mathrm{m}$ long; longer cell-free lanes result in the cessation of wave propagation. The time needed for the reappearance of the $\mathrm{Ca}^{2+}$ at the distal astrocytic culture increases as the lane length increases. However, the calculated velocity across the cell-free lanes is similar to the velocity through regions of confluent astrocytic networks. Furthermore, the probability of crossing a cell-free lane correlates with the length of the lane but not with the distance from the stimulation site where the $\mathrm{Ca}^{2+}$ wave was first evoked. ${ }^{27}$

Finally, it has been suggested that abnormal wave propagation in astrocytic tissues is linked to pathological conditions such as spreading depression, migraine, ${ }^{3,28}$ and epilepsy. ${ }^{29}$ Specifically in epilepsy, disruption of the normal brain function results from intensive burst activity from groups of neurons. Several recent studies have shown that astrocytes may play a key role in this disease. ${ }^{29-33}$ 
Motivated by these phenomena, in a previous study ${ }^{34}$ we presented a minimal signal transduction model that was able to account for wave propagation in homogeneous astrocytic networks. Furthermore, we investigated the effect of chemistry on the properties of wave propagation, and we found that it can dramatically affect the range of propagation. However, astrocytic networks are hardly homogeneous. Thus, the model could not simulate wave blocking and crossing phenomena arising from the existence of cell-free lanes or injured tissue, both of which are sources of domain inhomogeneity. Moreover, astrocytic networks are inherently discrete since cells can be viewed as localized ATP sources separated by regions where ATP degrades in the extracellular space. Such geometric effects were not modeled previously, on account of the homogeneity assumption. Thus, a quantitative understanding of the effects of spatial inhomogeneities on the characteristics of wave propagation and blocking in astrocytic cellular networks could offer insights into the role of astrocytes in mammalian brain function as well as several astrocyte-related pathological conditions.

The problem of modeling wave propagation in inhomogeneous media has been the focus of many previous efforts. Using simple caricature models, more detailed neuronal transduction models, or models describing $\mathrm{Ca}^{2+}$ dynamics, several researchers have demonstrated complex propagation patterns resulting from spatial inhomogeneities.

Goldstein and Rall $^{35}$ presented a reaction-diffusion model to study the effect of changing core conductor geometry in an axon. They demonstrated changes in shape and velocity of the wave as well as blocking and reflection phenomena. Pauwellusen ${ }^{36}$ employed a generalization of the Goldstein-Rall model and studied propagation in the FitzHugh-Nagumo equations when the cross-sectional area of the axon increases abruptly, thereby increasing the phenomenological diffusion coefficient. Furthermore, Pauwellusen showed that a step increase in the axon's diameter can result in wave blocking. Rinzel ${ }^{37}$ simulated reflections (echo waves) and blocking phenomena resulting from inhomogeneities along the excitable medium, due to either geometrical change or altered cell coupling. Extending Pauwellusen's and Rinzel's work, Zhou and Bell ${ }^{38}$ showed that wave reflections are observed if there is a sufficient delay in the propagation of the wave in the thick part of the axon. Thus, the thin part of the axon has already undergone the refractory period and a backwards propagating (echo) wave is formed.

Poptsova and Guria ${ }^{39}$ considered a different problem in which inhomogeneities arise from the existence of regions of decreased excitability, which can also be considered as a source of domain inhomogeneity. Such regions are playing the role of barriers with "height" that depends on the degree of excitability suppression. For this case, they studied wave tunneling for a bistable and an excitable medium, modeled by the Nagumo and the FitzHugh-Nagumo equations, respectively. ${ }^{40,41}$ They demonstrated wave blocking and computed the critical length and "height" of the barrier that results in cessation of wave-front or pulse propagation.

Furthermore, Sneyd and Sherratt ${ }^{42}$ investigated the case in which inhomogeneity in an excitable medium arises from the existence of nonexcitable regions (gaps) rather than changes in diffusion coefficient or decreased excitabilities. They derived explicit formulas for the critical length of a single gap that blocks wave propagation in an excitable medium described by one equation with a cubic-shaped reaction term. By extending their analysis to blocking from a single gap for the Li-Rinzel model ${ }^{43}$ for $\mathrm{Ca}^{2+}$ dynamics, they showed that higher excitability levels result in lower critical gap lengths. Furthermore, they investigated the effect of placing multiple gaps on the propagation of waves in the oscillatory regime of a $\mathrm{Ca}^{2+}$ dynamics model by Atri et al. ${ }^{44}$ which was originally developed for $\mathrm{Ca}^{2+}$ waves in the Xenopus laevis oocyte. For this case, Sneyd and Sherratt showed that the geometry can affect the speed of the resulting waves.

Phase plane techniques have also been employed to explain wave blocking in generic excitable systems. Lewis and Keener ${ }^{45}$ presented a geometrical method for the computation of the critical gap length for blocking, which is also useful in characterizing the stability of the steady-state solutions. Yang et $a l .{ }^{46}$ extended this analysis to multiple gaps and showed that in the case in which a large number of gaps exist, propagation of wave fronts can be facilitated. In addition, Aronson et $a l^{47}$ obtained analytical solutions for the piecewise linear McKean model in a configuration comprised of an arbitrary number of gaps. Equations for the critical gap length that results in blocking at the $M$ th gap were derived by investigating the conditions for the existence and stability of standing wave solutions. Furthermore, in agreement to Yang et al., ${ }^{46}$ it was shown that propagation of a wave front can be facilitated for a large number of gaps. More specifically, if an arrangement of $N$ identical equidistant gaps blocks the wave at the $M$ th gap, then it can be observed that an arrangement of $K>N$ gaps blocks the wavefront at the $L$ th gap with $L>M$ (same distance between gaps as previously). Finally, Aronson et al. ${ }^{47}$ derived a condition that links the level of excitability with the number of gaps for the occurrence of this phenomenon.

The aforementioned studies clearly demonstrated that the existence of spatial inhomogeneities in an excitable medium can greatly affect wave-propagation characteristics. Phenomena such as blocking and reflections were observed, as well as changes in the speed and the shape of the waves. These studies utilized simple caricature models, more detailed neuronal transduction models, or models of $\mathrm{Ca}^{2+} \mathrm{dy}-$ namics at the single-cell level. However, in order to model wave propagation in astrocytic networks, one has to take into account that astrocytes are stimulated with completely different mechanisms than neurons. Thus, our single-cell model has a different mathematical structure that takes into account the salient features of the astrocytic signal transduction pathway. Specifically, an extracellular messenger with nonexcitable dynamics (ATP) drives the excitable $\mathrm{Ca}^{2+}$ subsystem. This coupling will reveal several novel phenomena that can be useful in understanding wave propagation in inhomogeneous astrocytic networks.

The rest of the paper is organized as follows. We first present the mathematical formulation and comment on the numerical scheme used to solve the problem. We subsequently analyze the propagation patterns obtained for the 

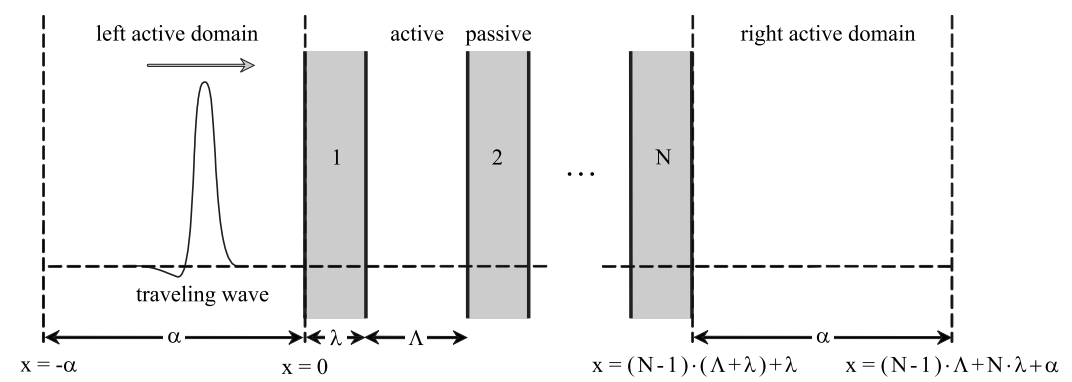

FIG. 1. Schematic illustration of the inhomogeneous domain. Passive areas are denoted as gray shaded blocks. A wave, coming from $-\infty$ (left active domain), meets a series of $N$ gaps of length $\lambda$ separated by $(N-1)$ active domains of length $\Lambda$.

one- and two-gap cases, and for the case of multiple gaps we demonstrate two propagation patterns of possible experimental significance. Finally, we discuss possible insights that this work can offer in understanding wave propagation and blocking in astrocytic cellular networks.

\section{MATHEMATICAL FORMULATION}

In order to study wave propagation in a spatially inhomogeneous astrocytic network, we use a reaction-diffusion formulation with no flux boundary conditions. The geometry of the network is shown in Fig. 1. To model inhomogeneities, we assume that there exist two types of regions. In the socalled active regions, diffusion as well as release-uptake and production-degradation reactions of species take place. In the so-called passive domains (or gaps), only diffusion and degradation of species in the extracellular space occur. Therefore, the two kinds of domains have different reaction terms and can also have different diffusion coefficients. Clusters of identical active and passive domains follow each other successively, as shown in Fig. 1, which depicts a wave that comes from $\mathrm{x}=-\alpha$ (left active domain) and meets a series of $N$ gaps of length $\lambda$ separated by $(N-1)$ active areas of length $\Lambda$. Our objective is to investigate the link between the singlecell signal transduction mechanisms, the geometric characteristics of the network (gap length and length of active regions), and the ability of such a wave to propagate through the inhomogeneous domain.

Let us define the set of all active domains as

$$
\begin{aligned}
\mathfrak{A}= & {[-\alpha, 0) \cup \bigcup_{\mathrm{i}=1}^{\mathrm{N}-1}[(\mathrm{i}-1) \cdot(\Lambda+\lambda)} \\
& +\lambda, \mathrm{i} \cdot(\Lambda+\lambda)) \cup \ldots \cup[(\mathrm{N}-1) \cdot(\Lambda+\lambda) \\
& +\lambda,(\mathrm{N}-1) \cdot(\Lambda+\lambda)+\lambda+\alpha]
\end{aligned}
$$

and the set of all passive domains as

$$
\mathfrak{P}=\bigcup_{\mathrm{i}=1}^{\mathrm{N}}[(\mathrm{i}-1) \cdot(\Lambda+\lambda), \mathrm{i} \cdot(\Lambda+\lambda)+\lambda) .
$$

Let $\mathrm{u}_{\mathrm{w}}(\mathrm{x}, \mathrm{t})$ be the vector of all the species' concentrations, $\mathrm{D}_{\mathrm{Z}}$ be the diagonal matrix with the diffusion coefficients of the species at domain $Z$ ( $Z$ is either $\mathfrak{A}$ for active regions or $\mathfrak{P}$ for gaps), and let $\mathrm{f}_{Z}(\underset{\sim}{\mathrm{u}})$ be the vector that gives the reaction terms as a function of species concentrations in domain $Z$. Then the general reaction diffusion formulation is

$$
\begin{aligned}
& \frac{\partial \underset{\sim}{\mathrm{u}}}{\partial \mathrm{t}}=\underset{\sim}{\mathrm{D}_{\mathrm{A}}} \cdot \frac{\partial^{2} \underset{\sim}{\mathrm{u}}}{\partial \mathrm{x}^{2}}+{\underset{\sim}{\mathrm{A}}}_{\mathrm{A}}(\underset{\sim}{\mathrm{u}}) \quad \mathrm{x} \in \mathfrak{A},
\end{aligned}
$$

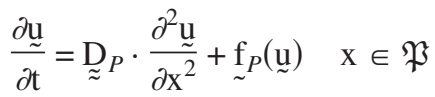

subject to the requirement for continuity of concentrations and fluxes for the diffusing species at the boundaries between the passive and active domains, and no flux conditions at the boundary of the astrocytic network. Thus, continuity of concentrations is imposed as

$$
\begin{aligned}
\left.\underset{\tilde{\sim}}{\mathrm{M}} \cdot \mathrm{u}\right|_{\mathrm{x}^{-}} & =\left.\underset{\tilde{\sim}}{\mathrm{M}} \cdot \mathrm{u}\right|_{\mathrm{x}^{+}} \quad \forall \mathrm{x}=(\mathrm{i}-1) \cdot(\Lambda+\lambda)+\mathrm{j} \cdot \lambda ; \\
\mathrm{i} & =1,2, \ldots, \mathrm{N} ; \quad \mathrm{j}=0,1,
\end{aligned}
$$

where $\underset{\sim}{\mathrm{M}}$ is defined as

$$
\underset{\tilde{\sim}}{\mathrm{M}_{\mathrm{i}, \mathrm{j}}}= \begin{cases}1 & \text { if } \underset{\tilde{\tilde{D}}}{\mathrm{D}} \mathrm{i}, \mathrm{j} \\ 0 & \text { otherwise. }\end{cases}
$$

Continuity of fluxes reads

$$
\begin{aligned}
& \left.\underset{\sim}{\mathrm{D}_{A}} \cdot \frac{\partial \underline{\mathrm{u}}}{\partial \mathrm{x}}\right|_{\mathrm{x}^{-}}=\left.\underset{\tilde{\sim}}{\mathrm{D}_{P}} \cdot \frac{\partial \underline{\mathrm{u}}}{\partial \mathrm{x}}\right|_{\mathrm{x}^{+}} \quad \forall \mathrm{x}=(\mathrm{i}-1) \cdot(\Lambda+\lambda) ; \\
& \mathrm{i}=1,2, \ldots, \mathrm{N} \\
& \left.\underset{\tilde{z}}{\mathrm{D}_{P}} \cdot \frac{\partial \underline{\mathrm{u}}}{\partial \mathrm{x}}\right|_{\mathrm{x}^{-}}=\left.\underset{\tilde{\sim}}{\mathrm{D}_{A}} \cdot \frac{\partial \underline{\mathrm{u}}}{\partial \mathrm{x}}\right|_{\mathrm{x}^{+}} \quad \forall \mathrm{x}=(\mathrm{i}-1) \cdot(\Lambda+\lambda)+\lambda ; \\
& \mathrm{i}=1,2, \ldots, \mathrm{N}
\end{aligned}
$$

and the no-flux boundary conditions are imposed at the outer left and right active domains as follows:

$$
\begin{aligned}
& \left.\underset{\sim}{\mathrm{D}_{A}} \cdot \frac{\partial \mathrm{u}}{\partial \mathrm{x}}\right|_{\mathrm{x}^{+}}=0 \quad \mathrm{x}=-\alpha, \\
& \left.\underset{\sim}{\mathrm{D}_{A}} \cdot \frac{\partial \underline{\mathrm{u}}}{\partial \mathrm{x}}\right|_{\mathrm{x}^{-}}=0 \quad \mathrm{x}=(\mathrm{N}-1) \cdot(\Lambda+\lambda)+\lambda+\alpha .
\end{aligned}
$$

Conditions (7) can be expressed as a single equation if the diffusion coefficients in the active and passive domains are the same. Furthermore, the initial condition is 


$$
\underset{\sim}{\mathrm{u}}(\mathrm{x}, 0)={\underset{\sim}{\mathrm{u}}}_{0}(\mathrm{x}) \quad \mathrm{x} \in \mathfrak{A} \cup \mathfrak{P} .
$$

To describe the single-cell signal transduction dynamics, we employed a previously developed model. ${ }^{34}$ The model consists of the Li-Rinzel ${ }^{43}$ equations that describe the release of $\mathrm{Ca}^{2+}$ from the endoplasmic reticulum, augmented with equations that account for ATP mediated $\mathrm{IP}_{3}$ production and ATP release to the extracellular space. Four species are considered: ATP, $\mathrm{IP}_{3}, \mathrm{Ca}^{2+}$, with concentrations denoted by $\mathrm{H}, \mathrm{I}, \mathrm{C}$, and $y$, a lumped variable that contains information about the fraction of open channels:

$$
\underset{\sim}{\mathrm{u}}=\left[\begin{array}{llll}
\mathrm{H} & \mathrm{I} & \mathrm{C} & y
\end{array}\right]^{T} .
$$

Clearly channels do not diffuse, nor does $y \cdot \mathrm{Ca}^{2+}$ has been experimentally found to have negligible contribution to astrocytic wave propagation due to buffering effects. ${ }^{48}$ Furthermore, $\mathrm{IP}_{3}$ diffuses slower than ATP and its diffusion was numerically found to have no qualitative effect and negligible quantitative effect on propagation phenomena in astrocytic cultures. ${ }^{34}$ Hence, we let only ATP diffuse, and for simplicity we assumed that it has the same diffusion coefficient in the active and passive domains,

$$
\underset{\approx}{\mathrm{D}_{A}}=\underset{\approx}{\mathrm{D}_{P}}=\left[\begin{array}{cccc}
\mathrm{D}_{\mathrm{ATP}} & 0 & 0 & 0 \\
0 & 0 & 0 & 0 \\
0 & 0 & 0 & 0 \\
0 & 0 & 0 & 0
\end{array}\right]
$$

The reaction terms for the active domains $\operatorname{are}^{34}$

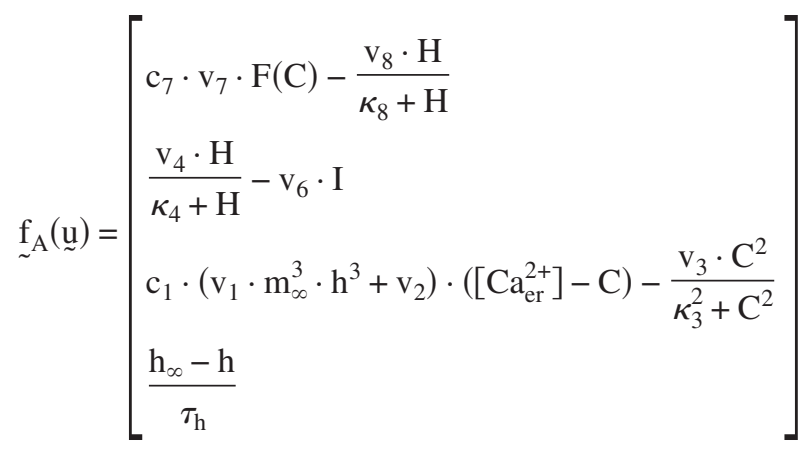

and for the passive domains,

$$
\underset{\sim}{\mathrm{f}_{P}}(\underset{\sim}{\mathrm{u}})=\left[\begin{array}{l}
-\theta \cdot \frac{\mathrm{v}_{8} \cdot \mathrm{H}}{\kappa_{8}+\mathrm{H}} \\
0 \\
0 \\
0
\end{array}\right],
$$

where

$$
\begin{array}{r}
F(C)=\frac{\frac{F_{0}}{F_{0}-1}-2 \cdot \frac{C}{C_{\max }}}{\frac{1}{F_{0}-1}-\left(\frac{C}{C_{\max }}\right)^{2}} \\
m_{\infty}=\left(\frac{I}{I+d_{1}}\right) \cdot\left(\frac{C}{C+d_{5}}\right),
\end{array}
$$

$$
\begin{aligned}
& {\left[\mathrm{Ca}_{\mathrm{er}}^{2+}\right]=\frac{\mathrm{c}_{0}-\mathrm{C}}{\mathrm{c}_{1}},} \\
& \tau_{\mathrm{h}}=\frac{1}{\mathrm{a}_{2} \cdot\left(\mathrm{Q}_{2}+\mathrm{C}\right)}, \\
& \mathrm{h}_{\infty}=\frac{\mathrm{Q}_{2}}{\mathrm{Q}_{2}+\mathrm{C}}, \\
& \mathrm{Q}_{2}=\mathrm{d}_{2} \frac{\mathrm{I}+\mathrm{d}_{1}}{\mathrm{I}+\mathrm{d}_{3}} .
\end{aligned}
$$

The parameters of these equations are given in Table I.

Moreover, the initial condition consists of flat equilibrium concentration profiles for all species except ATP, for which a square pulse in space is added as a perturbation. The amplitude of the pulse is $A$ and the width is $\delta$. The pulse is imposed at the left boundary of the inhomogeneous domain, $\mathrm{x}=-\alpha$. Thus, if $\mathrm{H}_{\mathrm{o}}, \mathrm{I}_{\mathrm{o}}, \mathrm{C}_{\mathrm{o}}$, and $\mathrm{h}_{\mathrm{o}}$ are the steady-state concentrations, then the initial condition is

$$
\underset{\sim}{\mathrm{u}_{0}}(\mathrm{x})=\left[\begin{array}{l}
\mathrm{H}_{\mathrm{o}}+\mathrm{A} \cdot[\Phi(\mathrm{x}+\alpha)-\Phi(\mathrm{x}+\alpha-\delta)] \\
\mathrm{I}_{\mathrm{o}} \\
\mathrm{C}_{\mathrm{o}} \\
\mathrm{h}_{\mathrm{o}}
\end{array}\right],
$$

where $\Phi$ denotes the Heaviside step function. A key property of the single-cell-signal transduction model is excitability. As demonstrated in Fig. 2(a), an excitable system exhibits a sharp response for stimuli above a threshold. In the singlecell simulations depicted, a square pulse in time is imposed on ATP. The pulse has duration $1 \mathrm{~s}$ and variable amplitude; the stimulus imposed is equal to the product of amplitude and duration. We observe that an ATP stimulus of $2.9 \mu \mathrm{M}$ produces a minor response. However, a stimulus of $3.0 \mu \mathrm{M}$ is above the threshold and results in a disproportionately large departure from the steady state. This behavior is due to the excitable properties of the $\mathrm{Ca}^{2+}$ dynamics, which exert a positive feedback on ATP. It is important to note that the ATP dynamics are not inherently excitable, whereas those of intracellular $\mathrm{Ca}^{2+}$ are. It is the coupling between the two subsystems $\left(\mathrm{ATP} / \mathrm{IP}_{3}\right.$ and $\left.\mathrm{Ca}^{2+}\right)$ through the positive feedback from $\mathrm{Ca}^{2+}$ to the ATP release that renders the overall signal transduction system excitable. ${ }^{34}$

Figure 2(b) shows that the level of excitability is strongly affected by the $\mathrm{IP}_{3}$ degradation rate $\left(\mathrm{v}_{6}\right)$. Specifically, the plot shows the critical amplitude resulting in excitable response for various degradation rates. Apparently, for higher excitability levels, the system can exhibit excitable responses for lower pulse amplitudes (stimuli). Furthermore, we observe that lower $\mathrm{IP}_{3}$ degradation rates $\left(\mathrm{v}_{6}\right)$ result in higher excitabilities up to the point that the system starts to exhibit oscillatory response (for $\mathrm{v}_{6}=4.854 \mathrm{~s}^{-1}$ ). Thus, in the simulations that will follow, the effect of excitability level was quantified by the values of the $\mathrm{IP}_{3}$ degradation rate.

For the numerical solution of Eqs. (1)-(20), a mixed numerical scheme was developed, since some of the species diffuse and others remain localized. Space discretization and time integration were used for the diffusing species (ATP), 
TABLE I. Parameters of the model.

\begin{tabular}{|c|c|c|c|}
\hline Symbol & Value & Units & Description \\
\hline $\mathrm{c}_{0}$ & 2.0 & $\mu \mathrm{M}$ & total $\mathrm{Ca}^{2+}$ concentration \\
\hline $\mathrm{c}_{1}$ & 0.185 & (dim/less) & ratio: ER volume vs cytoplasm volume \\
\hline $\mathrm{v}_{1}$ & 6.0 & $\mathrm{~s}^{-1}$ & channel flux constant \\
\hline $\mathrm{v}_{2}$ & 0.11 & $\mathrm{~s}^{-1}$ & $\mathrm{Ca}^{2+}$ leak flux constant \\
\hline $\mathrm{v}_{3}$ & 0.9 & $\mu \mathrm{M} \mathrm{s}^{-1}$ & maximum $\mathrm{Ca}^{2+}$ pump flux \\
\hline$\kappa_{3}$ & 0.05 & $\mu \mathrm{M}$ & constant in Hill function \\
\hline$a_{2}$ & 0.2 & $(\mu \mathrm{M} \mathrm{s})^{-1}$ & $\begin{array}{l}\text { rate constant for } \mathrm{Ca}^{2+} \text { binding in inhibitory site } \\
\text { presence } \mathrm{IP}_{3}\end{array}$ \\
\hline $\mathrm{d}_{1}$ & 0.13 & $\mu \mathrm{M}$ & dissociation constant \\
\hline $\mathrm{d}_{2}$ & 1.049 & $\mu \mathrm{M}$ & dissociation constant \\
\hline $\mathrm{d}_{3}$ & 0.9434 & $\mu \mathrm{M}$ & dissociation constant \\
\hline$d_{5}$ & 0.08234 & $\mu \mathrm{M}$ & dissociation constant \\
\hline $\mathrm{v}_{4}$ & 4.0 & $\mu \mathrm{M} \mathrm{s}^{-1}$ & maximum $\mathrm{IP}_{3}$ production rate \\
\hline$\kappa_{4}$ & 0.3 & $\mu \mathrm{M}$ & constant in Michaelis-Menten func. for $\mathrm{H}^{\mathrm{a}}$ \\
\hline $\mathrm{v}_{6}$ & 4.95 & $\mathrm{~s}^{-1}$ & $\mathrm{IP}_{3}$ degradation rate constant $\mathrm{b}^{\mathrm{b}}$ \\
\hline $\mathrm{c}_{7}$ & 1.0 & (dim/less) & ratio: cytoplasmic vs extracellular volume \\
\hline $\mathrm{v}_{7}$ & 5.0 & $\mu \mathrm{M} \mathrm{s}^{-1}$ & maximum ATP production rate \\
\hline $\mathrm{F}_{0}$ & 0.05 & (dim/less) & leak ATP secretion (when $\left.\left[\mathrm{Ca}^{2+}\right]=0 \mu \mathrm{M}\right)$ \\
\hline $\mathrm{C}_{\max }$ & 1.5 & $\mu \mathrm{M}$ & $\mathrm{Ca}^{2+}$ concentration maximizing ATP feedback ${ }^{\mathrm{c}}$ \\
\hline $\mathrm{v}_{8}$ & 6.0 & $\mu \mathrm{M} \mathrm{s}^{-1}$ & maximum ATP degradation rate \\
\hline$\kappa_{8}$ & 5.0 & $\mu \mathrm{M}$ & $\begin{array}{l}\text { constant in Michaelis-Menten function for ATP } \\
\text { degradation }\end{array}$ \\
\hline$\theta$ & 0 & $(\operatorname{dim} / l e s s)$ & ratio: ATP degrad. rate in passive vs active region \\
\hline $\mathrm{D}_{\mathrm{ATP}}$ & 350 & $\mu \mathrm{M}^{2} \mathrm{~s}^{-1}$ & ATP diffusion coefficient ${ }^{\mathrm{d}}$ \\
\hline
\end{tabular}

${ }^{\mathrm{a}}$ From Kastritsis et al. (Ref. 53) and Charest et al. (Ref. 54), the Michaelis-Menten constant is about $-\log ([\mathrm{ATP}])=6.5$ thus $10^{-6.5} \approx 0.3 \mu \mathrm{M}$.

${ }^{\mathrm{b}}$ Range of values for this parameter can be found in Wang et al. (Ref. 55).

${ }^{c}$ This value for $\mathrm{C}_{\max }$ is a representative maximum value for intracellular $\mathrm{Ca}^{2+}$ concentrations that have been experimentally observed.

${ }^{\mathrm{d}} D_{\mathrm{ATP}}=330-500 \mu \mathrm{m}^{2} / \mathrm{s}$ at $20-37^{\circ} \mathrm{C}$ (Refs. 10 and 56).

whereas for each of the nondiffusing species, an ordinary differential equation was solved for each node by the time integrator.

Specifically, the Finite Element Method $\left(\mathrm{FEM}^{49}\right)$ was chosen for the discretization in space. The local definition of the basis functions and the weighted residual formulation offers the ability to naturally satisfy the interfacial conditions (continuity of fluxes and concentrations) without any additional approximations. Quadratic basis functions were used because they strike the best balance between accuracy and numerical stability, thereby maximizing the computational efficiency of the algorithm. Simulations with progressively finer discretizations were performed up to the point that the solution does not change. The system of linear equations that arises after discretization was solved by using subroutines bandec and banbks, ${ }^{50}$ which exploit the banded structure of the mass matrix.

The fully implicit Adams Moulton second order was considered as the time integrator because of its numerical stability properties. Yet, it was found to yield inaccurate results for large time steps and requires the solution of a nonlinear system at each time step. Thus, it is more efficient to use an explicit scheme instead. The Runge-Kutta fourthorder method (RK4) was chosen ${ }^{51}$ due to its high accuracy and numerical stability as well as its time explicit nature. However, the key benefit of RK4 is that it provides accurate solutions while using time steps close to the numerical stability limit. Trial simulations performed with smaller time steps were found to yield the same results. This is particularly important for the problem under consideration, where different complex propagation patterns exist in neighboring regimes of parameter space.

Finally, the mixed numerical scheme described is consistent with the original system of equations, prevents the appearance of artifacts in the solution ("wiggly" profiles), and for the case of the conditionally stable time integrator, it makes efficient use of the stability range.

\section{RESULTS}

Waves of infinite range are predicted by the model for parameter values rendering the single-cell dynamics excitable, when the domain is homogeneous. ${ }^{34}$ In this section, we will investigate the effects of domain inhomogeneities as well as parameters affecting excitability at the single-cell level on the overall characteristics of the predicted wave patterns. Traveling waves will be shown in the form of contour plots on the $(\mathrm{x}, \mathrm{t})$ space.

\section{One-gap case}

Consider the simplest case in which a single nonexcitable gap is placed in the astrocytic network. Figure 3(a) 


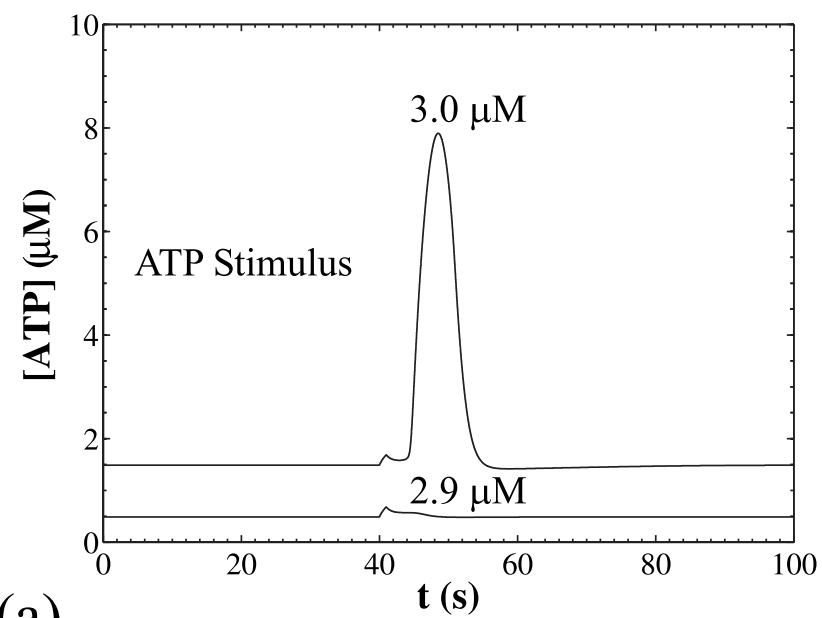

(a)

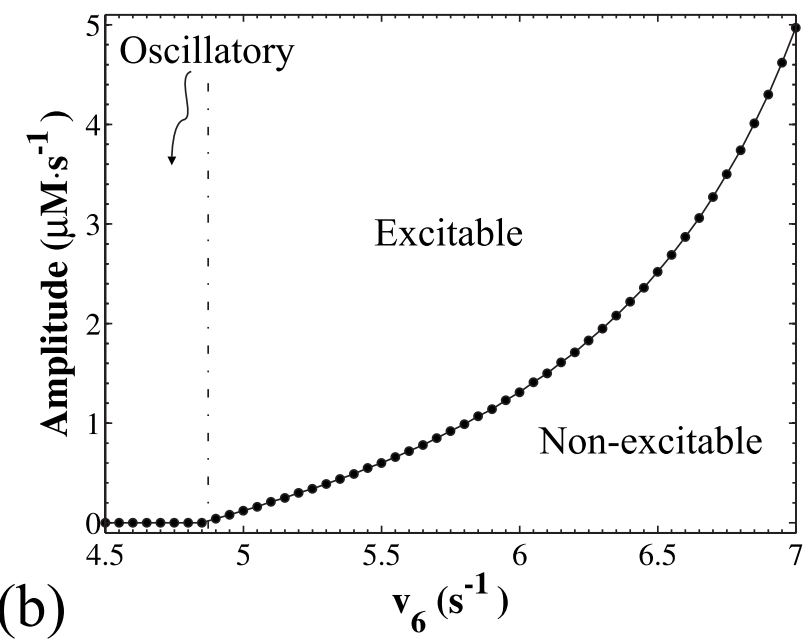

FIG. 2. (a) Excitable and nonexcitable response: the single-cell signal transduction model is initialized from the unique steady state corresponding to $\mathrm{v}_{6}=5.2 \mathrm{~s}^{-1}$ and a square pulse in time is imposed on ATP at $\mathrm{t}=40 \mathrm{~s}$, with duration $1 \mathrm{~s}$. The subthreshold amplitude, $0.29 \mu \mathrm{M} / \mathrm{s}$ (stimulus =duration-amplitude), produces a small departure from the steady state, whereas the superthreshold amplitude, $0.30 \mu \mathrm{M} / \mathrm{s}$, produces a sharp response indicative of excitability. The excitable time course has been shifted $+1 \mu \mathrm{M}$ for clarity. (b) Excitability vs $\mathrm{IP}_{3}$ degradation rate $\left(\mathrm{v}_{6}\right)$. Critical amplitudes for pulse duration $1 \mathrm{~s}$ and various excitability levels as quantified by the $\mathrm{IP}_{3}$ degradation rate $\mathrm{v}_{6}$. The separatrix of oscillatory and excitable behavior occurs for $\mathrm{v}_{6}=4.854 \mathrm{~s}^{-1}$. Other parameters as in Table I.

shows an ATP-Ca ${ }^{2+}$ wave, which is initiated in the left excitable regime and surpasses a small gap as a result of the ATP diffusion. When the wave reaches the gap, it ceases temporarily, since the passive region does not permit further propagation. However, ATP diffuses through the gap to the following active region, thereby making possible the regeneration of the wave therein. Thus, the excitable wave surpasses the gap, a phenomenon that has also been experimentally observed. ${ }^{27}$

Gaps with lengths slightly larger than those required for unidirectional wave crossing generate a rather interesting effect. As shown in Fig. 3(b), "reflected" waves are continuously emitted from the gap, which exhibits pacemaker behavior. This phenomenon can be explained as follows: when the wave reaches the gap, ATP diffuses toward the right active region, which leads to wave regeneration there. This regeneration results in a transient increase of the ATP con- centration at the boundary between the gap and the right active region. Consequently, ATP diffuses backwards and triggers the regeneration of a reflected wave moving into the left active region. This phenomenon is repeated, so that sequences of two-way reflected waves are continuously emitted from the gap.

It is interesting to observe that regeneration was not possible in the previous case in which the gap length was smaller. In fact, regeneration is only possible if the astrocytes of the left region have undergone the refractory phase and are able to be excited again by the backwards diffusing ATP. Thus, two factors play a significant role in the generation of reflected waves. First, the gap needs to be sufficiently large in order to delay the crossing of the wave, so that the cells are given the time necessary to undergo the refractory period. This is why reflection is not observed for smaller gap lengths [Fig. 3(a)]. Second, high levels of excitability facilitate the reexcitation of astrocytes lying in the left active region. Low excitability levels would render impossible the reexcitation by the small amounts of backwards diffusing ATP and thus reflection patterns would not be observed.

Nevertheless, gaps of even larger lengths [Fig. 3(c)] block the wave completely because insufficient ATP diffuses through the gap in order to excite the astrocytes of the right excitable regime. ATP wave blocking has been experimentally observed ${ }^{27}$ and it is also intuitively expected. Clearly, the more excitable the astrocytic network is, the larger the gaps that the ATP wave can cross.

More complicated patterns can be observed for very high excitability levels, for which the system is close to the oscillatory regime. Such a pattern, referred to as a backwards reflection pattern, is shown in Fig. 3(d). In this case, the refractory period is markedly small; as a result, the astrocytes at the right end of the left active region can quickly become reexcited by the ATP concentrated in the passive region. Thus, the passive regime acts as an ATP buffer, which is replenished transiently after every reexcitation of the astrocytes. This process results in an infinite sequence of backwards traveling ATP-Ca ${ }^{2+}$ pulses. The large gap length, however, does not permit sufficient ATP to pass through and excite the cells at the far end of the passive region, as shown in Fig. 3(e), in which the concentrations of the two "edges" of the gap are plotted as functions of time.

For the highly excitable regime, two-way reflection patterns can also be observed for gap lengths lower than those generating backwards reflections. However, for sufficiently large gaps, these patterns are qualitatively different from those observed for lower excitability levels, as shown in Fig. 4. The difference lies in the fact that for such high excitability levels, each "edge" of the passive regime acts as an individual oscillator. The two oscillators are coupled due to the ATP diffusion, a phenomenon that can disrupt the one-to-one correspondence of pulses emitted in each direction, left and right. Thus, more complex wave emission patterns can be observed such as the 13:14 (number of pulses emitted to the left versus to the right) shown in Fig. 4(a). This is not possible for lower excitabilities for which the two edges emit waves successively [Fig. 4(b)] due to the cooperation necessary to emit waves in the first place. 

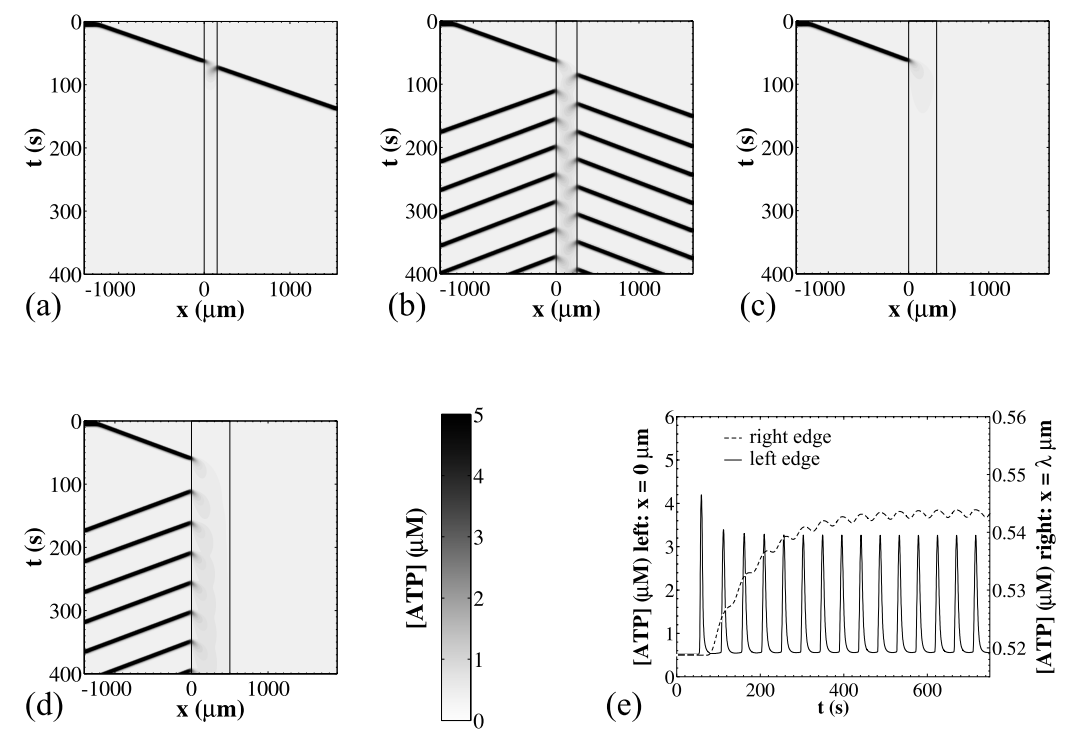

FIG. 3. (a) A wave generated in the left excitable regime surpasses a gap of length $150 \mu \mathrm{m}$. (b) A larger gap, of length $250 \mu \mathrm{m}$, generates reflected waves. (c) An even larger gap $(350 \mu \mathrm{m})$ can block the wave. Parameters as in Table I, except $\mathrm{v}_{6}=4.95 \mathrm{~s}^{-1}$ for panels (a), (b), and (c). (d) Backwards reflection pattern generated in the highly excitable regime $\left(\mathrm{v}_{6}=4.95 \mathrm{~s}^{-1}, \lambda=500 \mu \mathrm{m}\right)$. (e) Time course of ATP concentration for both "edges" of the gap. Parameters for panels (d) and (e) as in Table I, except $\mathrm{v}_{6}=4.9 \mathrm{~s}^{-1}$. Negligible degradation of ATP in the gap was assumed in all four simulations.

Thus, we see that different propagation patterns are exhibited as a function of the excitability level and the geometric parameters of the astrocytic network. Figure 5(a) displays the critical curves separating the main classes of the propagation patterns obtained for the one-gap case, assuming negligible [Fig. 5(a)] and non-negligible [Fig. 5(b)] ATP degradation in the gap. As also shown in Fig. 2, higher $\mathrm{IP}_{3}$ degradation rates result in lower excitabilities. On the other hand, the geometric parameter for the one-gap case is the length of the gap $(\lambda)$. The curves presented in the $\lambda-\mathrm{v}_{6}$ diagram are essentially borderlines between regions where dif-

(a)


FIG. 4. (a) Asymmetric two-way reflection pattern for high excitability levels: the ratio of number of pulses emitted from the left edge vs the right edge of the gap (1:r) is equal to 13:14. Parameters as in Table I, except $\mathrm{v}_{6}=4.9 \mathrm{~s}^{-1}$, gap length $340 \mu \mathrm{m}$. (b) Symmetric reflection pattern for slightly lower excitability levels: the ratio (1:r) now is 1:1. Parameters as in Table I, except $\mathrm{v}_{6}=4.95 \mathrm{~s}^{-1}$, gap length $260 \mu \mathrm{m}$. ferent patterns are exhibited. In the lower region, the wave is not blocked by the gap; in the upper region, the wave is blocked; and in the small triangular area, reflected waves are observed. For highly excitable single-cell dynamics (e.g.,

(a)
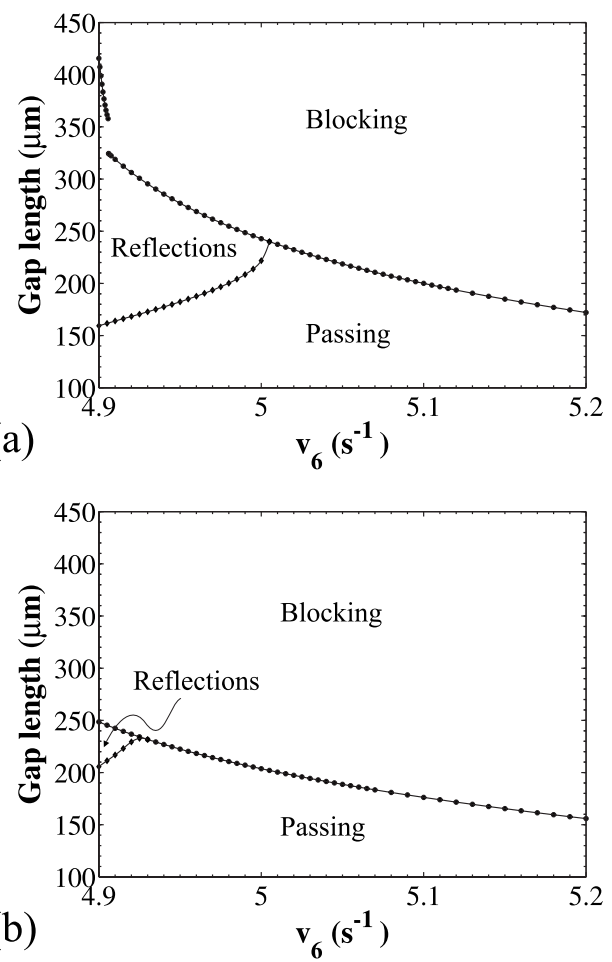

FIG. 5. Critical curves separating the main classes of the observed patterns in the one-gap case. (a) Effect of excitability, as quantified by the $\mathrm{IP}_{3}$ degradation rate $\left(\mathrm{v}_{6}\right)$, on the existence of propagation patterns in the case of negligible ATP degradation in the gap $(\theta=0$, all parameters as in Table I). The discontinuity at $\mathrm{v}_{6} \approx 4.906 \mathrm{~s}^{-1}$ corresponds to the emergence of the backwards reflections pattern, observed in the blocking regime above the bulleted line. (b) Effect of non-negligible ATP degradation in the gap $(\theta=0.01$, all other parameters as in Table I $)$. 

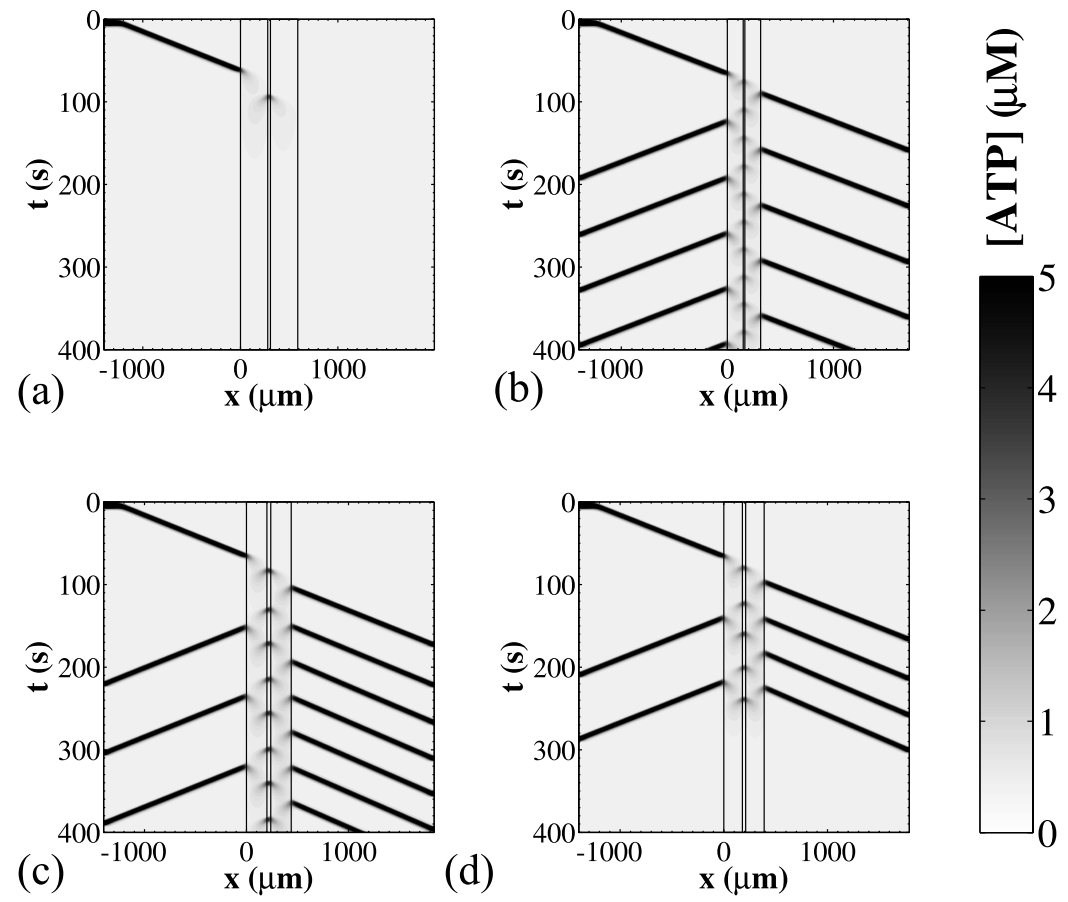

FIG. 6. Patterns observed in the two-gap case: Blocking in the second gap [(a), $\lambda=280 \mu \mathrm{m}, \Lambda=30 \mu \mathrm{m}]$. Stable symmetric [(b), $\lambda=150 \mu \mathrm{m}, \Lambda=15 \mu \mathrm{m}]$ and asymmetric [(c), $\lambda=200 \mu \mathrm{m}, \Lambda=40 \mu \mathrm{m}]$ reflection patterns as well as transient [(d), $\lambda=180 \mu \mathrm{m}, \Lambda=30 \mu \mathrm{m}]$ ones can also be observed in the two-gap case. Parameters as in Table I (negligible ATP degradation) except $\mathrm{v}_{6}=4.95 \mathrm{~s}^{-1}$ for (a) and $\mathrm{v}_{6}=5.02 \mathrm{~s}^{-1}$ for (b), (c), and (d).

$\mathrm{v}_{6}=4.95 \mathrm{~s}^{-1}$ ), as the gap length increases, the behavior of the system undergoes a transition from the pattern where the wave crosses the gap, to wave reflection, and then, for larger gap lengths, to wave blocking. For lower levels of excitability, the reflected wave pattern is no longer exhibited.

Furthermore, notice that the critical line that separates blocking from propagation patterns is monotonically decreasing. This is due to the fact that less excitable astrocytes require higher ATP concentrations to be excited. Smaller gaps transiently allow for higher ATP amounts to diffuse through. As a result, lower excitability levels require smaller gaps for the network to exhibit the wave-crossing pattern. On the other hand, the critical curve that separates blocking from reflection is monotonically increasing. This can be attributed to the time required to reexcite the cells of the left active region (refractory period). Less excitable cells require more time and thus larger gaps.

By comparing the results in Figs. 5(a) and 5(b), we observe that ATP degradation has a dual effect on the critical curves. First, all the curves are shifted to lower gap lengths. This is reasonable, since ATP degradation results in a loss of ATP during its transport through the gap, which is no longer purely diffusive. Thus, in order to observe the same patterns as before, smaller gap lengths are required to allow for sufficient ATP amounts to reach the right active regime. Second, the region where reflections are observed shrinks [Fig. 5(b)]. This effect can be attributed to the losses of the backwards diffusing ATP resulting from degradation. Thus, smaller amounts of ATP are reaching the astrocytes of the left active regime. Subsequently, for reexcitation and thus reflection, higher excitability levels are required.

\section{Two-gap case}

In the two-gap case, the same patterns as in the one-gap case can be generated, namely crossing the two gaps and blocking at the first gap. However, due to the presence of the intermediate active region, more complex patterns are also predicted by the model.

Such a pattern is shown in Fig. 6(a), in which blocking occurs at the second gap, after the wave has crossed the first gap. It is interesting to note that for gap length $\lambda=280 \mu \mathrm{m}$, crossing of the first of the two gaps is observed, even though it is greater than the critical gap length that permits the crossing of a single gap placed alone at the astrocytic network $(\lambda=276.8 \mu \mathrm{m})$ for the same excitability level $\left(\mathrm{v}_{6}=4.95 \mathrm{~s}^{-1}\right)$. In this sense, the two gaps act synergistically with the active domain in order to allow the wave to cross the first gap.

This counterintuitive behavior can be explained as follows. Due to the excitable characteristics of the single-cell single transduction dynamics, when the ATP concentration is below the excitability threshold, the decrease in its concentration within an active region is more significant than its decrease as a result of pure diffusion within a passive domain. Thus, the gap length required to block the wave in the first gap will be smaller in the case in which the gap borders with an active region (one-gap configuration).

Furthermore, reflection patterns also exist in the two-gap case [Figs. 6(b)-6(d)]. Of course, in parameter regions where a single gap can generate reflections, it is reasonable that two gaps can also do so. However, it turns out that two gaps can generate reflections in regions of the parameter space where a single gap is unable to do so, such as for sufficiently low 


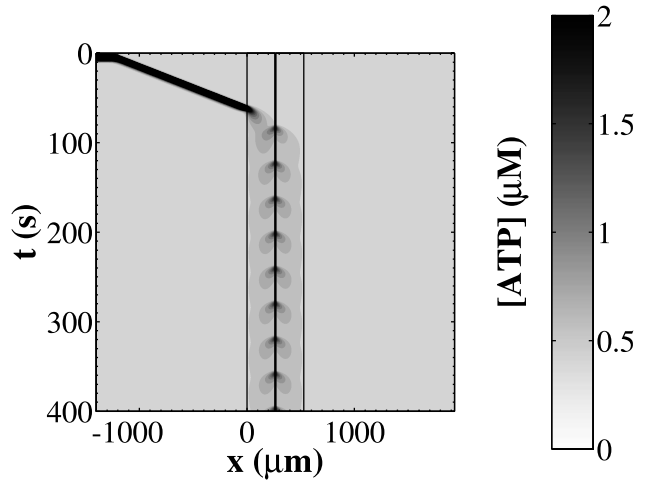

FIG. 7. Trapping pattern $(\lambda=260 \mu \mathrm{m}, \Lambda=10 \mu \mathrm{m})$. Parameters as in Table I (negligible ATP degradation) except $\mathrm{v}_{6}=4.95 \mathrm{~s}^{-1}$.

excitability levels (see Fig. 5). Such a pattern is shown in Fig. 6(b). In this case, the existence of the intermediate active area acts as a relay transmitter and the existence of two gaps provides a greater lag necessary for the cells of the left and right active regions to undergo the refractory phase. Symmetric [Fig. 6(b), see also animation in supplemental information $\left.{ }^{57}\right]$ as well as asymmetric reflection patterns can be observed [Fig. 6(c)]. In the latter case, the distal active region generates two reflected waves for one wave generated in the proximal active region. Furthermore, transient simulation patterns can also be observed. Such a pattern is shown in Fig. 6(d), in which the intermediate active region fires prematurely at some point in time. This firing results in the cessation of the reflections, since the left and right active regions are still in the refractory period and cannot be excited.

Finally, a novel "trapping" pattern is shown in Fig. 7 (see also animation in supplemental information ${ }^{57}$ ). In this case, once the cells of the intermediate active region are excited, they release ATP that temporarily accumulates in the two gaps, which act as buffers. The amount of ATP is not sufficient to excite the cells of the left or the right active regions. However, it can excite the cells of the intermediate active region, thus creating a sequence of pulses trapped in the intermediate active regime. The simulations show that reexcitation of the astrocytes of the left or right active region is impossible even in the long run. This phenomenon can be attributed to two factors: (i) The left and right active regions suffer greater ATP losses than the intermediate active region does, because they face the purely diffusive gap only on one side; on the other side, they extend to infinity. (ii) The ATP pulses that are trapped in the intermediate active region are not large enough. Thus, they never reach ATP concentrations as high as those observed for pulses generated in an infinitely large active region. As a consequence, the pulses remain "trapped" in the intermediate active region. Note that for the parameter set of this simulation, a single isolated cell would be unable to generate autonomous oscillations; it would only exhibit excitable behavior.

Figure 8 displays the critical curves separating the main classes of the observed patterns. The diagram discriminates only between blocking and two-way reflection patterns. The critical gap length is plotted as a function of the intermediate active region length (x axis). Figures 7(a)-7(c) correspond to progressively decreasing excitability levels. Notice that all curves converge to some characteristic values as the active area length tends to infinity. This phenomenon is due to the fact that gaps that are too far apart behave like solitary gaps; there is no interaction between them. Consequently, these characteristic values are the single gap critical lengths. For example, in Fig. 8(a) the excitability level is high enough for a single gap to exhibit reflections. Thus, for the two-gap case, the critical curve that separates reflections from cross-
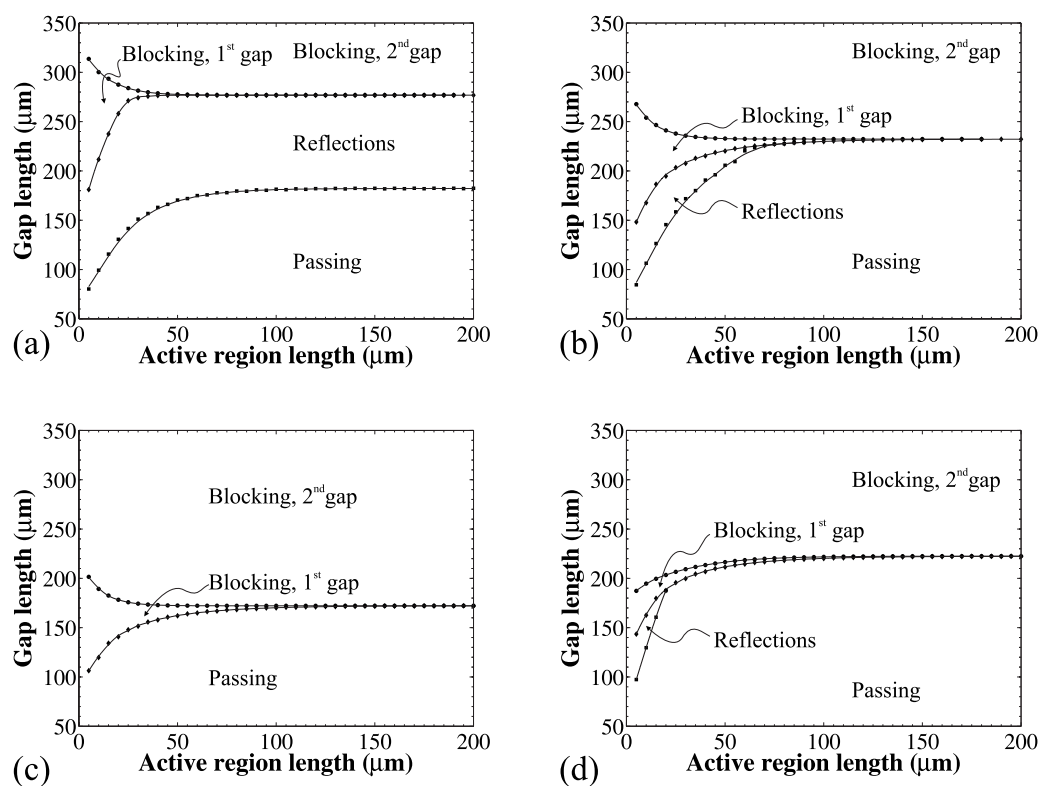

FIG. 8. Critical curves separating the main classes of the observed patterns in the two-gap case. (a) Critical regions for $\mathrm{v}_{6}=4.95 \mathrm{~s}^{-1}$ : passing, reflections, and blocking at any of the two gaps can be observed. (b),(c) Effect of lower excitability levels: $\mathrm{v}_{6}=5.02 \mathrm{~s}^{-1}$ for panel (b) and $\mathrm{v}_{6}=5.20 \mathrm{~s}^{-1}$ for panel (c). Negligible ATP degradation assumed $(\theta=0)$ for panels (a), (b), and (c). (d) Effect of non-negligible ATP degradation in the gaps $\left(\theta=0.01, \mathrm{v}_{6}=4.95 \mathrm{~s}^{-1}\right)$. Other parameters as in Table I. 

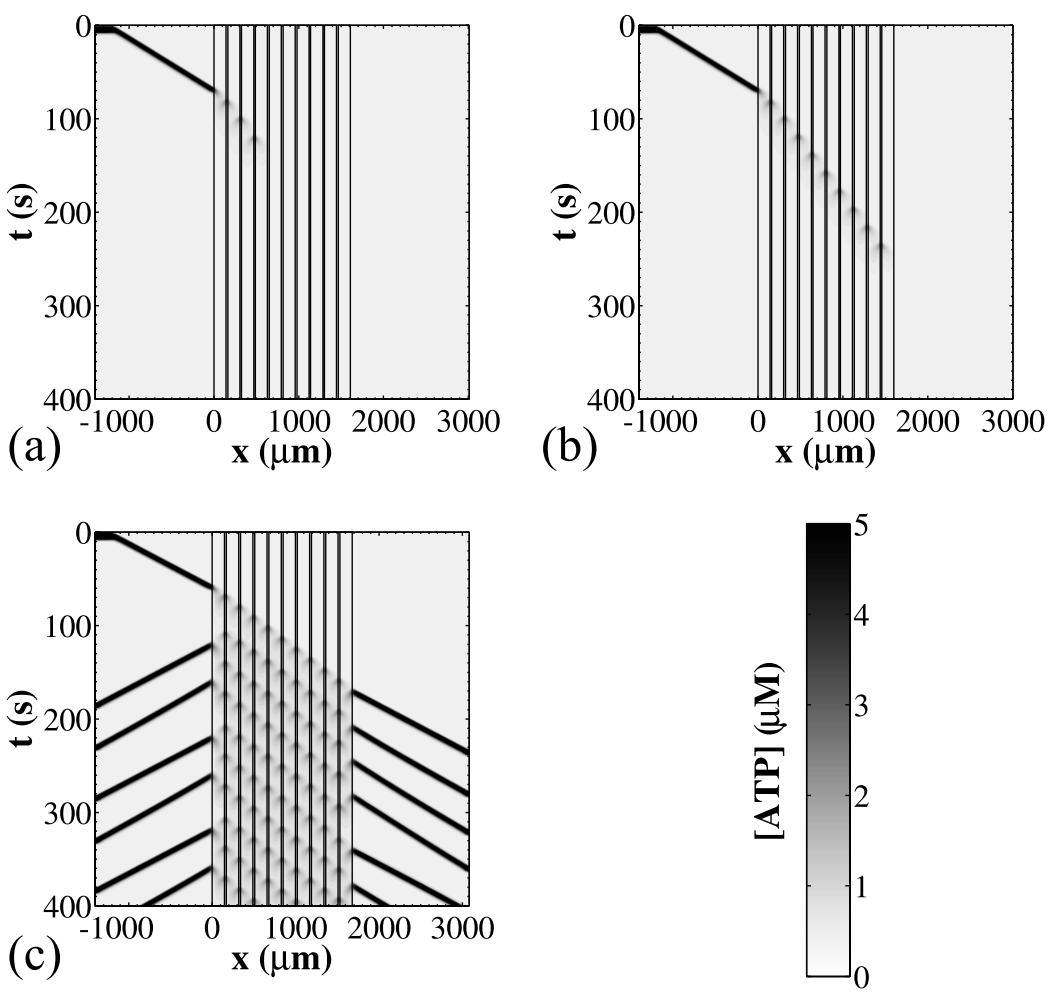

FIG. 9. (a),(b) Wave propagation on ten-gap arrangements with the same active region length $(\Lambda=20 \mu \mathrm{m})$ but variable gap lengths: wave blocking can occur at any of the gaps, for example at the third $[(\mathrm{a}), \lambda=143 \mu \mathrm{m}]$ or the tenth $[(\mathrm{b}), \lambda=142 \mu \mathrm{m}] . \mathrm{v}_{6}=5.2 \mathrm{~s}^{-1}$ for panels (a) and (b). (c) Reflection pattern in a 10-gap arrangement $\left(\lambda=150 \mu \mathrm{m}, \Lambda=20 \mu \mathrm{m}, \mathrm{v}_{6}=4.95 \mathrm{~s}^{-1}\right)$. Other parameters as in Table I.

ing the first gap approaches the critical single gap length that separates reflections from passing at the same excitability level. Similarly, the critical curve that separates blocking at the first versus the second gap approaches the critical single gap blocking length.

Notice that the curve that separates blocking at the first versus the second gap is monotonically decreasing. In the case of purely diffusive gaps, both gaps act synergistically, thereby facilitating the crossing of the wave through the first gap. For increasing lengths of the active region, this synergy progressively diminishes and thus the critical curve appears monotonically decreasing. ${ }^{47}$

Furthermore, as shown in Fig. 8(b), in the purely diffusive two-gap case, reflections can be observed for excitability levels lower than those required for reflections in the one-gap case. The reflections are observed for gaps that are (i) smaller than those required for passing through the first gap in the one-gap case and (ii) separated by a sufficiently small active region. Naturally, reflections are suppressed for large lengths of the intermediate region. As a matter of fact, the critical curve separating reflections from blocking is monotonically increasing, and as the length of the active region increases, it merges with the critical curve that delimits the regime of blocking at the first gap.

Finally, for low excitabilities the only possible patterns are blocking at the first or the second gap, as shown in Fig. 8 (c). The monotonicity of the critical curves is maintained and the limiting value they both approach is the critical length for blocking in the one-gap case.
ATP degradation in the gaps changes the monotonicity of the critical curve separating blocking at the first versus the second gap. Figure 8(d) shows that the critical curve separating the two blocking patterns is now monotonically increasing. This effect is due to the gaps now contributing to the ATP degradation. Thus, for smaller active regimes they act synergistically in degrading the ATP rather than preserving it, as in the case of a purely diffusive gap. Consequently, the critical gap length for crossing the first of the two gaps will be smaller than the critical length for crossing a single gap.

\section{Multiple-gap case}

The case of multiple gaps placed in the astrocytic network resembles astrocytes in a row separated by cell-free regions. Even though the geometry of a real astrocytic network is higher dimensional, the patterns exhibited in the onedimensional multiple-gap case can offer valuable insight into understanding experimental findings.

Specifically, two blocking patterns are shown in Figs. 9(a) and 9(b). The only difference between panels (a) and (b) is the gap length, and it is apparent that wave propagation ceases at a different gap. This observation implies that wave blocking can occur after a specific number of gaps have been passed; in other words, the range of propagation depends strongly on the geometry of the astrocytic network. Consequently, blocking patterns can be observed as a result of the inhomogeneous nature of the astrocytic network emanating 
from the different degrees of confluency in a cell culture. Experimental observations suggest that wave blocking indeed occurs at typical ranges of 36 cell diameters (or a radial distance of $180 \mu \mathrm{m})^{5}$

It needs to be noted, though, that if the wave is blocked at the $N$ th gap, the sensitivity of the critical gap length increases with respect to $N$. In other words, the difference between the critical gap lengths for blocking at the first versus the second gap is larger that the difference between the critical gap lengths for blocking at the ninth versus the tenth gap. In general, the range of gap lengths that allow for propagation through several cell diameters followed by blocking is small. This effect was observed in our simulations, where we have identical gaps and active regions; however, it is not expected to be observed in astrocytic networks with random configurations. In such networks, the existence of a large gap after several small gaps will create a "bottleneck" in the propagation of waves and will eventually lead to blocking for a larger range of gap lengths. This assertion needs to be investigated further, since in this study we were not concerned with wave propagation in randomly configured astrocytic networks.

Furthermore, notice that the speed with which the wave travels in the cluster of gaps is smaller than the speed observed in the left active domain [Fig. 9(b)]. This effect is due to the crossings of the gaps, which generate small pauses in the propagation of the wave. Such pauses have been experimentally observed when the $\mathrm{Ca}^{2+}$ wave reaches the boundaries of the cells. ${ }^{27}$ Moreover, the experimentally observed variations of the wave speed ${ }^{27}$ can also be partially attributed to the confluency of the astrocytic cultures. The more confluent the astrocytic culture, the faster the wave, as predicted by the simulation.

Complex reflection patterns can also be observed in the multiple-gap case, as shown in Fig. 9(c) for an inhomogeneous domain consisting of 10 gaps. The wave from the left active regime enters the cluster of gaps and generates reflected waves that travel through the cluster to both the outer left and right active domains. For smaller lengths of active regions, the amount of ATP regenerated within the cells is not enough for the wave to escape the inhomogeneous domain. Hence, two-way reflections cease to exist and yield (as in the two-gap case) a "trapping" pattern, where all active areas between the gaps are excited continuously and asynchronously, thus generating waves that cannot extend beyond the boundaries of the inhomogenous domain (see also supplemental material ${ }^{57}$ for a movie of a representative simulation). The asynchronous nature of ATP and $\mathrm{Ca}^{2+}$ regeneration within the cells makes the wave pattern almost look as if the cells within the inhomogeneous domain "fire" stochastically.

This effect, in turn, resembles the experimentally observed ATP release by point source bursts. ${ }^{52}$ Since our model is fully deterministic, this result indicates that certain geometries of the astrocytic network may result in a continuous and asynchronous (but ordered) reverberation of the signal within a cluster of cells. Thus, bursting by point sources may not be an entirely random phenomenon, due to the stochastic nature of the intracellular reaction events, as implied in the cited paper. Yet the fact that ATP is released by only $0.1-1.0 \%$ of the total cell population within a 6 -min time interval, and the random spatial distribution of bursts, is not consistent with our simulation results, which show frequent ordered bursts throughout the cell population. It is possible that random placement of gaps in the astrocytic network can affect the bursting patterns, yet such an effect was not investigated in this study.

Continuous reverberation of the signal also resembles brain function during a seizure, when a group of neurons exhibits intensive burst activity. It may be possible that astrocytes play an important role in initializing and/or maintaining the seizure-prone state of the brain. Our numerical results show that continuous reverberation is facilitated by higher excitabilities and a large number of relatively small gaps. Therefore, it would be interesting to perform experiments to test for reverberation patterns in astrocytic cultures with different degrees of confluency.

\section{DISCUSSION}

We investigated the effects of spatial inhomogeneities in astrocytic cellular networks. For the description of individual cell behavior, we used a model accounting for ATP-mediated stimulation of intracellular $\mathrm{Ca}^{2+}$ dynamics and ATP release in the extracellular space. Spatial inhomogeneities, arising from the different degrees of confluency of an astrocytic culture or from traumatic or ischemic tissue, were modeled as domains where intracellular reactions do not occur (passive domains, gaps). Depending on the geometry of the network, the ATP wave was able to cross such domains or was halted by them. Complex propagation patterns such as reflections were observed.

Specifically for the one-gap case, for low excitability levels, the only possible patterns are wave blocking or crossing of the gap. Such patterns have also been experimentally observed. ${ }^{27}$ The critical gap length separating these patterns is a monotonically decreasing function of excitability. This result agrees with intuition, which suggests that the easier it is to excite the network, the larger the gaps that the resulting waves can cross. Furthermore, for higher excitability levels, two-way reflections can be observed as a result of a continuous forward and backward diffusion of ATP in the gap. The reflection pattern is observed for sufficiently large gaps, which give the astrocytes the time necessary to recover between subsequent excitations of the ATP that diffuses through the gap. Finally, a backwards reflection pattern was observed due to ATP buffering in the gap. The inclusion of degradation in the gap shifts the critical blocking gap lengths to lower values and shrinks the reflective regime.

For the case of two gaps separated by an intermediate active domain, blocking can occur either at the first or at the second gap. The critical gap length that separates these two blocking patterns is a decreasing function of the intermediate active domain length for purely diffusive gaps. This result shows that the second gap facilitates the crossing of the wave through the first gap, since the second gap diminishes the overall degradation of ATP, thereby enabling the excitation of the intermediate active domain. However, if the ATP degradation in the gaps is not negligible, the gaps will contribute 
to ATP degradation rather than preservation. Thus, the critical gap length in this case is an increasing function of the length of the intermediate active domain. Furthermore, reflection patterns can be generated by the two-gap case too, and it was found that profound asymmetries can be observed for these reflections, in the sense that the ratio of pulses emitted in the right versus in the left direction was demonstrated to vary for different active and passive domain lengths.

Moreover, for the multiple gap case we demonstrated that blocking can occur at any gap depending on the length of the passive domain for a fixed active domain length. Thus, geometrical characteristics of the astrocytic network, such as the degree of confluency, may play an important role in modulating the propagation range of the $\mathrm{Ca}^{2+}$ wave. The experimentally observed blocking of the $\mathrm{Ca}^{2+}$ after typically 18 cell diameters ${ }^{5}$ may partially be a result of the geometry of the astrocytic network. Moreover, complex reflection patterns in the multiple gap case resemble the burst ATP-release patterns, which were experimentally observed in astrocytic cultures. ${ }^{52}$ Thus, the presence of inhomogeneities may have a causal role on this phenomenon.

The reflective patterns observed in all cases may offer insight into wave propagation behavior in pathological conditions such as epilepsy. In such conditions, an area of lower activity in the brain is continuously emitting waves acting as a pacemaker. Therefore, a mechanism that could explain this behavior is the continuous forward and backward diffusion of the messenger in the damaged tissue, a phenomenon that disrupts the unidirectional mode of wave propagation and creates this pacemaker effect. Such effects cannot be predicted by generic excitable models that do not take into account the salient features of the underlying biological processes. Thus, the model analyzed may offer valuable insight into formulating testable hypotheses for the abnormal wave propagation in pathological situations.

${ }^{1}$ P. Brodal, The Central Nervous System (Oxford University Press, New York, 1998).

${ }^{2}$ P. G. Haydon, Nat. Rev. Neurosci. 2, 185 (2001)

${ }^{3}$ M. Nedergaard, B. Ransom, and S. A. Goldman, Trends Neurosci. 26, 523 (2003).

${ }^{4}$ M. L. Cotrina, J. H.-C. Lin, A. Alves-Rodrigues, S. Liu, J. Li, H. AzmiGhadimi, J. Kang, C. C.G. Naus, and M. Nedergaard, Proc. Natl. Acad. Sci. U.S.A. 95, 15735 (1998)

${ }^{5}$ P. B. Guthrie, J. Knappenberger, M. Segal, M. V. L. Bennett, A. C. Charles, and S. B. Kater, J. Neurosci. 19, 520 (1999).

${ }^{6}$ W. T. Kim, M. G. Rioult, and A. H. Cornell-Bell, Glia 11, 173 (1994).

${ }^{7}$ A. H. Cornell-Bell and S. M. Finkbeiner, Cell Calcium 12, 185 (1991).

${ }^{8}$ A. H. Cornell-Bell, S. M. Finkbeiner, M. S. Cooper, and S. J. Smith, Science 247, 470 (1990).

${ }^{9}$ S. Finkbeiner, Neuron 8, 1101 (1992)

${ }^{10}$ E. A. Newman, J. Neurosci. 21, 2215 (2001).

${ }^{11}$ E. A. Newman and K. R. Zahs, Science 275, 844 (1997).

${ }^{12}$ A. C. Charles, Dev. Neurosci. (Basel, Switz.) 16, 196 (1994).

${ }^{13}$ A. C. Charles, E. R. Dirksen, J. E. Merril, and M. J. Sanderson, Neuron 6, 983 (1991).

${ }^{14}$ A. C. Charles, E. R. Dirksen, J. E. Merrill, and M. J. Sanderson, Glia 7, 134 (1993).

${ }^{15}$ A. C. Charles, C. C. Naus, D. Zhu, G. M. Kidder, E. R. Dirksen, and M. J. Sanderson, J. Biol. Chem. 118, 195 (1992).
${ }^{16}$ M. O. Enkvist and K. D. McCarthy, J. Neurochem. 59, 519 (1992).

${ }^{17}$ M. Takeda, D. J. Nelson, and B. Soliven, Glia 14, 225 (1995).

${ }^{18}$ L. Venance, N. Stella, J. Glowinski, and C. Giaume, J. Neurosci. 17, 1981 (1997).

${ }^{19}$ V. Venance, D. Piomelli, J. Glowinski, and C. Giaume, Nature (London) 376, 590 (1995).

${ }^{20}$ J. W. Dani, A. Chernjavsky, and S. J. Smith, Neuron 8, 429 (1992).

${ }^{21}$ V. Parpura, T. A. Basarsky, F. Liu, K. Jeftinija, S. Jeftinija, and P. G. Haydon, Nature (London) 369, 744 (1994).

${ }^{22}$ T. D. Hassinger, P. B. Atkinson, G. J. Strecker, L. R. Whalen, D. F. E., A. H. Koseel, and S. B. Kater, J. Neurobiol. 28, 159 (1995).

${ }^{23}$ B. Innocenti, V. Parpura, and P. G. Haydon, J. Neurosci. 20, 1800 (2000).

${ }^{24}$ S. V. Yagodin, L. Holtzclaw, and J. T. Russell, Mol. Cell. Biochem. 149, 137 (1995).

${ }^{25}$ S. V. Yagodin, L. Holtzclaw, C. A. Sheppard, and J. T. Russell, J. Neurobiol. 25, 265 (1994).

${ }^{26}$ A. Charles, Glia 24, 39 (1998).

${ }^{27}$ T. D. Hassinger, P. B. Guthrie, P. B. Atkinson, M. V. L. Bennett, and S. B. Kater, Proc. Natl. Acad. Sci. U.S.A. 93, 13268 (1996).

${ }^{28}$ D. H. Leibowitz, Proc. R. Soc. B 250, 287 (1992).

${ }^{29}$ R. D"Ambrosio, Physica A 103, 95 (2004).

${ }^{30}$ S. H. Lee, S. Magge, D. D. Spencer, H. Sontheimer, and A. H. CornellBell, Glia 15, 195 (1995).

${ }^{31}$ M. Khurgel and G. O. Ivy, Epilepsy Res. 26, 163 (1996).

${ }^{32}$ M. Vessal, C. B. Dugani, D. A. Solomon, W. M. Burnham, and G. O. Ivy, Brain Res. 1044, 190 (2005).

${ }^{33}$ G.-F. Tian, H. Azmi, T. Takano, Q. Xu, W. Peng, J. Lin, N. Oberheim, N. Lou, X. Wang, H. R. Zielke, J. Kang, and M. Nedergaard, Nat. Med. 11, 973 (2005).

${ }^{34}$ M. Stamatakis and N. V. Mantzaris, J. Theor. Biol. 241, 649 (2006).

${ }^{35}$ S. S. Goldstein and W. Rall, Biophys. J. 14, 731 (1974).

${ }^{36}$ J. P. Pauwelussen, Physica D 4, 67 (1981).

${ }^{37}$ J. Rinzel, Ann. N.Y. Acad. Sci. 591, 51 (1990).

${ }^{38}$ Y. Zhou and J. Bell, Math. Biosci. 119, 169 (1994).

${ }^{39}$ M. S. Poptsova and G. T. Guria, Gen. Physiol. Biophys. 16, 241 (1997).

${ }^{40} \mathrm{G}$. Ehrenstein and R. FitzHugh, Ionic Channels in Cells and Model Systems (Plenum Press, New York, 1986).

${ }^{41}$ J. S. Nagumo, S. Arimoto, and S. Yoshizawa, Proc. IRE 50, 2061 (1962).

${ }^{42}$ J. Sneyd and J. Sherratt, SIAM J. Appl. Math. 57, 73 (1997).

${ }^{43}$ Y.-X. Li and J. Rinzel, J. Theor. Biol. 166, 461 (1994).

${ }^{44}$ A. Atri, J. Amundson, D. Clapham, and J. Sneyd, Biophys. J. 65, 1727 (1993).

${ }^{45}$ T. J. Lewis and J. P. Keener, SIAM J. Appl. Math. 61, 293 (2000).

${ }^{46}$ J. Yang, S. Kalliadasis, J. H. Merkin, and S. K. Scott, SIAM J. Appl. Math. 63, 485 (2002).

${ }^{47}$ D. G. Aronson, N. V. Mantzaris, and H. G. Othmer, Discrete Contin. Dyn. Syst. 13, 843 (2005)

${ }^{48}$ N. L. Allbritton, T. Meyer, and L. Stryer, Science 258, 1812 (1992).

${ }^{49}$ J. N. Reddy, An Introduction to the Finite Element Method (Mc GrawHill, New York, 1993).

${ }^{50}$ W. H. Press, S. A. Teukolsky, W. T. Vetterling, and B. P. Flannery, Numerical Recipes in Fortran 77 (Cambridge University Press, Cambridge, UK, 1997).

${ }^{51}$ G. Dahlquist and A. Bjorck, Numerical Methods (Prentice-Hall, Englewood Cliffs, NJ, 1974).

${ }^{52}$ G. Arcuino, J. H.-C. Lin, T. Takano, C. Liu, L. Jiang, Q. Gao, J. Kang, and M. Nedergaard, Proc. Natl. Acad. Sci. U.S.A. 99, 9840 (2002).

${ }^{53}$ C. H. Kastritsis, A. K. Salm, and K. McCarthy, J. Neurochem. 58, 1277 (1992).

${ }^{54}$ R. Charest, V. Prpic, J. H. Exton, and P. F. Blackmore, Biochem. J. 227, 79 (1985).

${ }^{55}$ S. S. H. Wang, A. A. Alousi, and S. H. Thomson, J. Gen. Physiol. 105, 149 (1995).

${ }^{56}$ R. A. d. Graaf, A. v. Kranenburg, and K. Nicolay, Biophys. J. 78, 1657 (2000).

${ }^{57}$ See EPAPS Document No. E-CHAOEH-17-014703 for three movies illustrating the wave propagation patterns described and discussed in the text. This document can be reached through a direct link in the online article's HTML reference section or via the EPAPS homepage (http:// wwww.aip.org/pubservs/epaps.html). 